\title{
Non-compartment model to compartment model pharmacokinetics transformation meta-analysis - a multivariate nonlinear mixed model
}

\author{
Zhiping Wang ${ }^{1 \dagger}$, Seongho Kim ${ }^{1 \dagger}$, Sara K Quinney ${ }^{1}$, Jihao Zhou², Lang $\mathrm{Li}^{1^{*}}$ \\ From The ISIBM International Joint Conferences on Bioinformatics, Systems Biology and Intelligent \\ Computing (IJCBS) \\ Shanghai, China. 3-8 August 2009
}

\begin{abstract}
Background: To fulfill the model based drug development, the very first step is usually a model establishment from published literatures. Pharmacokinetics model is the central piece of model based drug development. This paper proposed an important approach to transform published non-compartment model pharmacokinetics (PK) parameters into compartment model PK parameters. This meta-analysis was performed with a multivariate nonlinear mixed model. A conditional first-order linearization approach was developed for statistical estimation and inference.
\end{abstract}

Results: Using MDZ as an example, we showed that this approach successfully transformed 6 non-compartment model PK parameters from 10 publications into 5 compartment model PK parameters. In simulation studies, we showed that this multivariate nonlinear mixed model had little relative bias $(<1 \%)$ in estimating compartment model PK parameters if all non-compartment PK parameters were reported in every study. If there missing noncompartment PK parameters existed in some published literatures, the relative bias of compartment model PK parameter was still small $(<3 \%)$. The $95 \%$ coverage probabilities of these PK parameter estimates were above $85 \%$.

Conclusions: This non-compartment model PK parameter transformation into compartment model meta-analysis approach possesses valid statistical inference. It can be routinely used for model based drug development.

\section{Background}

In recent decades, a new drug requires an average of 15 years and approaching a billion dollars in research and development [1]. Unfortunately, only one in 10 drugs that enter clinical testing receives eventual FDA approval $[2,3]$. Scientists have become increasingly mechanistic in their approach to drug development [4]. The recent ability to integrate genetic mutations and altered protein expression to pharmacokinetics (PK) and pharmacodynamic (PD) models allow a deeper understanding of the mechanisms of disease and therapies that are genuinely targeted [5-8]. In 2004, the FDA

\footnotetext{
* Correspondence: lali@iupui.edu

† Contributed equally

'Division of Biostatistics, Department of Medicine, School of Medicine, Indiana University, Indianapolis, IN, 46032, USA
}

released a report entitled: "Innovation or Stagnation, Challenge and Opportunity on the Critical Path to New Medical Products" [9]. Among its six general topic areas, three of them emphasized the importance of computational modeling and bioinformatics in biomarker development and streamlining clinical trials $[10,11]$. In multiple follow-up papers, clinical researchers, experimental biologists, computational biologists, and biostatisticians from both academia and industry all supported the FDA leadership in this critical path, and pointed out the challenges and opportunities of the PK/PD model based approach in drug development [12][13-15].

Pharmacokinetics model is the central piece of model based drug development. Almost all of the published PK data were summarized without fitting a compartment model. They are usually called non-compartment model 
PK parameters. For example, area under the concentration curve (AUC) is calculated from drug plasma concentration data based on trapezoid-rule [16]; clearance is calculated from dose and AUC; Cmax and Tmax are calculated from concentrations and their associated time points; terminal half-life is usually calculated from the last two to four sampling time-points directly; and etc. All these parameters cannot be used directly in a compartment model, and their transformation to compartment model PK parameters is essential.

\section{Methods}

\section{Non-Compartment Model to One-Compartment Model Transformation}

When a drug follows a one-compartment model of oral dose (1), the following non-compartment model PK parameters, $\mathbf{w}=\left(A U C, T_{\max }, T_{1 / 2}\right)$, are necessary to recover the one-compartment model parameters, $\beta=\left(k_{a}, k_{e}, V\right)$.

$$
\begin{aligned}
& f(\beta, t)=\frac{F \times \text { Dose } \times k_{a}}{V \times\left(k_{a}-k_{e}\right)}\left(e^{-k_{e} \times t}-e^{-k_{a} \times t}\right) . \\
& T_{1 / 2}=\frac{\log 2}{k_{e}}, \quad A U C=\frac{F \times \text { Dose }}{k_{e} \times V}, T_{\max }=\frac{\ln \left(k_{a}\right)-\ln \left(k_{e}\right)}{k_{a}-k_{e}},
\end{aligned}
$$

where, $F$ is an assumed known bioavailability, and dose denotes the oral dose. If however, only oral clearance, $C L_{p o}$ is reported, instead of $A U C$, then $C L_{p o}=V$ $\times K_{e}$. On the other hand, when dosing is through IV, only $\mathbf{w}=\left(\right.$ AUC, $\left.T_{1 / 2}\right)$, are necessary to recover the one compartment (3), with $\boldsymbol{\beta}=\left(k_{e}, V\right)$. The transformation formulas are defined in (4).

$$
\begin{aligned}
& f(\beta, t)=\frac{\text { Dose }}{V} \times e^{-k_{e} \times t} . \\
& T_{1 / 2}=\frac{\log 2}{k_{e}}, \quad A U C=\frac{\text { Dose }}{k_{e} \times V} .
\end{aligned}
$$

Similarly, if $C L_{i v}$ is reported, instead of $A U C$, then $C L_{I V}=V \times k_{e}$. These one-compartment-model and noncompartment model parameters and transformation were defined and discussed in great detail by [16].

\section{Non-Compartment Model to Two-Compartment Model Transformation}

If a drug's pharmacokinetics follows a two-compartment model with oral dose (5), the following non-compartment model PK parameters, $\mathbf{w}=\left(V d, A U C, T_{\max }, C L_{i v}\right.$, $\left.T_{1 / 2, \text { slow }}, T_{1 / 2, \text { fast }}\right)$, are necessary to recover the two-compartment model parameters, $\boldsymbol{\beta}=\left(k_{a}, k_{e}, V_{1}, k_{12}, k_{21}\right)$. Their transformations are defined in (6).

$$
\begin{aligned}
& f(\beta, t)=\frac{F \times \text { Dose } \times k_{a}}{V_{1}}\left[\begin{array}{l}
\frac{k_{21}-\lambda_{1}}{\left(k_{a}-\lambda_{1}\right)\left(\lambda_{2}-\lambda_{1}\right)} e^{-\lambda_{1} \times t}+\frac{k_{21}-\lambda_{2}}{\left(k_{21}-k_{a}\right)} \\
+\frac{\left.k_{a}-\lambda_{1}-\lambda_{2}\right)}{\left(\lambda_{1}-k_{a}\right)\left(\lambda_{2}-k_{a}\right)} e^{-\lambda_{2} \times t}
\end{array}\right] \\
& V d=\frac{k_{e} \times V_{1}}{\lambda_{2}}, A U C=\frac{F \times \text { Dose }}{k e \times V_{1}}, \\
& T_{\max }=\frac{\ln \left(k_{a}\right)-\ln \left(\lambda_{1}\right)}{k_{a}-\lambda_{1}}, C L_{i v}=k_{e} \times V_{1} \\
& T_{1 / 2, \text { slow }}=\frac{\log 2}{\lambda_{2}}, T_{1 / 2, \text { fast }}=\frac{\log 2}{\lambda_{1}} \\
& \text { where } \quad \lambda_{1}=1 / 2\left(\left(k_{12}+k_{21}+k_{e}\right)+\sqrt{\left(k_{12}+k_{21}+k_{e}\right)^{2}-4 k_{21} \times k_{e}}\right) \\
& \lambda_{2}=1 / 2\left(\left(k_{12}+k_{21}+k_{e}\right)-\sqrt{\left(k_{12}+k_{21}+k_{e}\right)^{2}-4 k_{21} \times k_{e}}\right) .
\end{aligned}
$$

If a drug's pharmacokinetics follows a two-compartment model with IV dose (7), the following non-compartment model PK parameters, $\mathbf{w}=\left(V d, A U C, C L_{i v}\right.$, $T_{1 / 2}$, slow, $\left.T_{1 / 2, \text { fast }}\right)$, are necessary to recover the twocompartment model parameters, $\boldsymbol{\beta}=\left(k_{e}, V_{1}, k_{12}, k_{21}\right)$. Their transformations are defined in (8).

$$
\begin{aligned}
& f(\beta, t)=\frac{\text { Dose }}{V_{1}}\left[\frac{k_{21}-\lambda_{1}}{\left(\lambda_{2}-\lambda_{1}\right)} e^{-\lambda_{1} \times t}+\frac{k_{21}-\lambda_{2}}{\left(\lambda_{1}-\lambda_{2}\right)} e^{-\lambda_{2} \times t}\right] \\
& \begin{array}{l}
V d=\frac{k_{e} \times V_{1}}{\lambda_{2}}, \text { AUC }=\frac{F \times \text { Dose }}{k e \times V_{1}}, C L_{i v}=k_{e} \times V_{1} \\
T_{1 / 2, \text { slow }}=\frac{\log 2}{\lambda_{2}}, T_{1 / 2, \text { fast }}=\frac{\log 2}{\lambda_{1}} \\
\text { where } \quad \lambda_{1}=1 / 2\left(\left(k_{12}+k_{21}+k_{e}\right)+\sqrt{\left(k_{12}+k_{21}+k_{e}\right)^{2}-4 k_{21} \times k_{e}}\right) \\
\lambda_{2}=1 / 2\left(\left(k_{12}+k_{21}+k_{e}\right)-\sqrt{\left(k_{12}+k_{21}+k_{e}\right)^{2}-4 k_{21} \times k_{e}}\right) .
\end{array}
\end{aligned}
$$

\section{A Multivariate Nonlinear Mixed Effect Model (Model Specification)}

Based on the multiple transformation equations between non-compartment model PK parameters and one or two compartment models, a multivariate nonlinear mixed effect model is established to estimate the population level PK parameters and their between study variances. Denote $w_{j k}$ as the observed $j$ th non-compartment PK parameter $\left(j=1, \ldots, J_{k}\right)$ from study $k(k=1, . ., K)$. Please note that not every study published all of the non-compartment parameters, hence $J_{k}$ varies from study to study. $\boldsymbol{\beta}_{k}$ is the study level compartment-model PK parameter vector, and $g_{j}\left(\boldsymbol{\beta}_{\boldsymbol{k}}\right)$ represents the transformation function. Because non-compartment model PK parameter, $w_{j k}$, is usually published in the form of a sample mean, model (9) shows that its variance is $\sigma_{j}^{2} / n_{k}$, where $\sigma_{j}^{2}$ is the within study variance (assumed to be homogeneous across studies), and $n_{k}$ is study $k$ sample size

$$
\begin{aligned}
& p\left(w_{j k} \mid \sigma_{j}^{2}, \beta_{k}\right)=N\left[g_{j}\left(\beta_{k}\right), \sigma_{j}^{2} / n_{k}\right], \\
& w_{j_{1} k} \perp w_{j_{2} k}, j_{1} \neq j_{2}, \quad\left(j_{1}, j_{2}\right) \subset(1, \ldots, J) .
\end{aligned}
$$

Model (9) also shows that the observed non-compartment model parameters, $\left(w_{1 k}, w_{2 k}, \ldots, w_{J_{k} k}\right)$, are 
independent. This is a multivariate nonlinear regression model.

Study level compartment model parameter $\boldsymbol{\beta}_{\boldsymbol{k}}$ is assumed to follow multivariate normal distribution (10), in which $\boldsymbol{\mu}$ is the population PK parameter vector and $\boldsymbol{\Omega}_{k}$ is its general covariance matrix.

$$
p\left(\beta_{k} \mid \Omega_{k}\right) \sim \operatorname{MVN}\left(\mu, \Omega_{k}\right)
$$

The joint likelihood of population/subject parameters and their covariance is shown in equation (11).

$$
\begin{aligned}
& L(\beta, \mu, \Omega, \Sigma ; \mathbf{w}, \mathrm{X}, \mathrm{Z}) \propto-\sum_{k=1}^{K} \sum_{j=1}^{I_{k}}\left[\frac{\left(w_{j k}-g_{j}\left(\beta_{k}\right)\right)^{2}}{2 \sigma_{j}^{2} / n_{k}}\right]-\sum_{k=1}^{K}\left[\frac{\left(\beta_{k}-\mu\right)^{T} \Omega_{k}^{-1}\left(\beta_{k}-\mu\right)}{2}\right] \\
& -\frac{\sum_{k=1}^{K} \log \left|\Omega_{k}\right|}{2}-\frac{\sum_{k=1}^{K} \sum_{j=1}^{I_{k}} \log \left|\sigma_{j}{ }^{2}\right|}{2} \\
& =-\frac{1}{2}\left\{[\mathrm{~W}-\mathrm{Xg}(\beta)]^{T} \Sigma^{-1}[\mathrm{~W}-\mathrm{Xg}(\beta)]+(\beta-\mathrm{Z} \mu)^{T} \Omega^{-1}(\beta-\mathrm{Z} \mu)+\log |\Omega|+\log |\Sigma|\right\},
\end{aligned}
$$

where $\mathbf{W}=\left(w_{11}, \ldots, w_{J_{K} K}\right)^{T}$ is a $\quad J \times 1 \quad\left(J=\sum_{k} J_{k}\right)$ observed non-compartment model PK parameter vector; $\mathbf{X}=\left(\mathbf{X}_{1}^{T}, \ldots, \mathbf{X}_{K}^{T}\right)^{T}$ is a $J_{\times} p$ indicator matrix, and $\mathbf{X}_{k}$ is a $J_{k} \times p$ matrix indicating the corresponding transformation function; $\mathbf{g}($.) is a $p \times 1$ transformation function vector; $\beta=\left(\beta_{1}^{T}, \beta_{2}^{T}, \ldots, \beta_{K}^{T}\right)^{T}$ is a study level compartmentmodel PK parameter vector; $\Sigma=\operatorname{diag}\left\{\Sigma_{1}, \ldots, \Sigma_{K}\right\}$ is a diagonal $J \times J$ covariance matrix for $\mathbf{W}$, and $\mathbf{Z}=\left(\mathbf{I}_{1}, \mathbf{I}_{2}, \ldots, \mathbf{I}_{K}\right)^{T} ; \mathbf{Z}=\left(\mathbf{I}_{1}, \mathbf{I}_{2}, \ldots, \mathbf{I}_{K}\right)^{T}$ is a $K p \times p$ design matrix relating study-specific parameter $\boldsymbol{\beta}$ to population parameter $\boldsymbol{\mu}$, and $\mathbf{I}_{k}$ is an identity matrix; and $\Omega=\operatorname{diag}\left\{\Omega_{1}, \ldots, \Omega_{K}\right\}$ is a $K p \times K p$ covariance matrix for study-specific parameter $\beta$.

This multivariate nonlinear mixed model (11) is different from the conventional univariate nonlinear mixed model [17] structurally in the additional design matrix $\mathbf{X}$ in front of the nonlinear function (i.e. transformation function $\mathbf{g}($.$) ). Model (11) is a meta-analysis approach,$ in which sample mean non-compartment model PK parameters are formulated. Among the existing nonlinear mixed model meta-analysis literatures, some dealt with the subject-level data from multiple studies $[18,19]$; the others dealt with sample mean drug concentration data $[20,21]$; and none of them discussed the meta-analysis on summarized PK parameters through the noncompartment model.

\section{A Multivariate Nonlinear Mixed Effect Model (Estimation and Inference)}

As a conditional first order linearization approach provides the least biased estimate in estimating the PK parameter with comparable efficiency [22,23]), it is chosen as the estimation approach for this multivariate nonlinear mixed model. This conditional first order linearization approach was firstly introduced by Lindstrom and Bates [24]. We revise their derivation based on our special meta-analysis multivariate nonlinear mixed model (11). This two-step estimation scheme is described as following.

Step 1: given the current estimate of variance component $\hat{\Omega}$ and $\hat{\Sigma}$, minimize the following objective function, $L_{1}$, with respect to $(\boldsymbol{\beta}, \boldsymbol{\mu})$.

$$
\begin{aligned}
L_{1}(\beta, \mu ; \hat{\Omega}, \hat{\Sigma}, \mathbf{W}, \mathbf{X}, \mathbf{Z})= & {[\mathrm{W}-\mathbf{X g}(\beta)]^{T} \hat{\Sigma}^{-1}[\mathrm{~W}-\mathbf{X g}(\beta)] } \\
& +(\beta-\mathbf{Z} \mu)^{T} \hat{\Omega}^{-1}(\beta-\mathbf{Z} \mu)
\end{aligned}
$$

Computationally, minimizing $L_{1}$ on $(\boldsymbol{\beta}, \boldsymbol{\mu})$ is an iterative process. Within each iteration, a linearization is applied to $\mathbf{X g}(\boldsymbol{\beta})$ with respect to $\boldsymbol{\beta}$, and a linear mixed model (13) is fitted [24].

$$
\begin{aligned}
& \tilde{\mathbf{W}}=\tilde{\mathbf{X}} \mu+\tilde{\mathbf{Z}} \mathbf{b}+\varepsilon, \quad \varepsilon \sim \operatorname{MNV}(0, \quad \Sigma), \\
& \tilde{\mathbf{X}}=\mathbf{X G}(\hat{\beta}) \mathbf{Z}, \quad \tilde{\mathbf{Z}}=\mathbf{X G}(\hat{\beta}), \quad \hat{\beta}=\hat{\mathbf{b}}+\mathbf{Z} \hat{\mu}, \\
& \tilde{\mathbf{W}}=[\mathbf{W}-\mathbf{X g}(\hat{\beta})+\tilde{\mathbf{X}} \hat{\mu}+\tilde{\mathbf{Z}} \hat{\mathbf{b}}], \quad \mathbf{G}_{\beta}(\hat{\beta})=\partial \mathbf{g}(\beta) /\left.\partial \beta\right|_{\hat{\beta}} .
\end{aligned}
$$

Parameters $(\boldsymbol{\mu}, \mathbf{b}, \boldsymbol{\beta})$ 's estimates and their covariance are

$$
\begin{aligned}
& {\left[\begin{array}{lc}
\tilde{\mathbf{X}}^{T} \hat{\Sigma}^{-1} \tilde{\mathbf{X}} & \tilde{\mathbf{X}}^{T} \hat{\Sigma}^{-1} \tilde{\mathbf{Z}} \\
\tilde{\mathbf{Z}}^{T} \hat{\Sigma}^{-1} \tilde{\mathbf{X}} & \tilde{\mathbf{Z}}^{T} \hat{\Sigma}^{-1} \tilde{\mathbf{Z}}+\Omega^{-1}
\end{array}\right]\left(\begin{array}{l}
\mu \\
\mathbf{b}
\end{array}\right)=\left[\begin{array}{c}
\tilde{\mathbf{X}}^{T} \hat{\Sigma}^{-1} \tilde{\mathbf{W}} \\
\tilde{\mathbf{Z}}^{T} \hat{\Sigma}^{-1} \tilde{\mathbf{W}}
\end{array}\right]} \\
& \hat{\mu}=\left(\tilde{\mathbf{X}}^{T} \hat{\mathbf{V}}^{-1} \tilde{\mathbf{X}}\right)^{-1} \tilde{\mathbf{X}}^{T} \hat{\mathbf{V}}^{-1} \tilde{\mathbf{W}}, \quad\left(\hat{\mathbf{V}}=\tilde{\mathbf{Z}} \hat{\mathbf{S}} \tilde{\mathbf{Z}}^{T}+\hat{\Sigma}\right), \\
& \hat{\mathbf{b}}=\hat{\Omega} \tilde{\mathbf{Z}}^{T} \hat{\mathbf{V}}^{-1}(\tilde{\mathbf{W}}-\tilde{\mathbf{X}} \hat{\mu}), \\
& \operatorname{cov}(\hat{\mu}, \hat{\mathbf{b}})=\left[\begin{array}{cc}
\tilde{\mathbf{X}}^{T} \hat{\Sigma} \hat{\Sigma}^{-1} \tilde{\mathbf{X}} & \tilde{\mathbf{X}}^{T} \hat{\Sigma} \hat{\Sigma}^{-1} \tilde{\mathbf{Z}} \\
\tilde{\mathbf{Z}}^{T} \hat{\Sigma}^{-1} \tilde{\mathbf{X}} & \tilde{\mathbf{Z}}^{T} \hat{\Sigma}^{-1} \tilde{\mathbf{Z}}+\Omega^{-1}
\end{array}\right]^{-1} . \\
& \operatorname{cov}(\hat{\beta})=\operatorname{cov}(\mathbf{Z} \hat{\mu}+\hat{\mathbf{b}})=(\mathbf{Z}, \mathbf{I}) \operatorname{cov}(\hat{\mu}, \hat{\mathbf{b}})(\mathbf{Z}, \mathbf{I})^{T}
\end{aligned}
$$

Step 2: given the current estimate, $(\hat{\beta}, \hat{\mu})$, minimize the following objective function, $L_{2}$, with respect to $\boldsymbol{\theta}$, which is the variance component parameter vector in $(\Omega, \Sigma)$, and it is of dimension $q$.

$$
\begin{aligned}
& L_{2}\left(\Omega, \sum ; \hat{\beta}, \hat{\mu}, \mathbf{W}, \tilde{\mathbf{X}}, \tilde{\mathbf{Z}}\right)=\mathbf{r}^{T} \mathbf{V}^{-1} \mathbf{r}^{T}+\log |\mathbf{V}|+\log \left|\tilde{\mathbf{X}}^{T} \mathbf{V}^{-1} \tilde{\mathbf{X}}\right|, \\
& \mathbf{r}=\mathbf{W}-\mathbf{X g}(\hat{\beta})+\tilde{\mathbf{Z}} \mathbf{b}, \mathbf{V}=\sum+\mathbf{Z} \Omega \tilde{Z}^{T} .
\end{aligned}
$$

This $L_{2}$ likelihood function is the restricted maximum likelihood for variance component estimates. The scores and the elements of information matrix for $\boldsymbol{\theta}$ are defined in (17).

$$
\begin{aligned}
& u_{s}=-\frac{1}{2} \operatorname{tr}\left(\mathbf{P} \frac{\partial \mathbf{V}}{\partial \theta_{s}}\right)+\frac{1}{2} \mathbf{r}^{T} \mathbf{V}^{-1} \frac{\partial \mathbf{V}}{\partial \theta_{s}} \mathbf{V}^{-1} \mathbf{r}=0, \\
& I(s, t)=\frac{1}{2} \operatorname{tr}\left(\mathbf{P} \frac{\partial \mathbf{V}}{\partial \theta_{s}} \mathbf{P} \frac{\partial \mathbf{V}}{\partial \theta_{t}}\right), \\
& \text { where, } 1 \leq s, t \leq q, \quad \mathbf{P}=\mathbf{V}^{-1}-\mathbf{V}^{-1} \tilde{\mathbf{X}}\left(\tilde{\mathbf{X}}^{T} \mathbf{V}^{-1} \tilde{\mathbf{X}}\right)^{-1} \tilde{\mathbf{X}}^{T} \mathbf{V}^{-1} .
\end{aligned}
$$


Hence, $\boldsymbol{\theta}$ can be estimated through an iterative Fisher algorithm. An alternative derivation of this two-step first order linearization is through a second order Laplace's approximation [25-27].

\section{Results}

Midazolam Non-Compartment Model Parameters to Compartment Model Parameters Transformation Data Analysis

After extensive literature search, 10 midazolam pharmacokinetics studies were identified, and their published non-compartment PK parameters are reported in Table 1. $\left(C_{\max }, A U C, T_{1 / 2 \text {,slow }}\right)$ were reported with high frequencies, i.e. 8 to 10 out 10 publications. $T_{1 / 2 \text {,fast }}$ was published only twice. Both $V_{d}$ and $C L_{i v}$ were published 5 to 6 times.

A multivariate nonlinear mixed effect model is fitted to these published non-compartment PK parameters to estimate their compartment model PK parameters. The NONMEM code is reported in Appendix I. In this meta-analysis, between study variances are assumed for $\left(V_{1}, k_{a}, k_{e}\right) .\left(k_{12}, k_{21}\right)$ were assumed to be the fixed effects across different studies without random effects, because only two papers published the MDZ distribution information, i.e. $T_{1 / 2 \text {,fast }}$. All of the non-compartment model parameters were log-transformed. They were assumed to have the same within study variance in log-scale (i.e. same coefficient of variance in the raw scale). All of the compartment model PK parameters were also log-transformed, and their between study standard deviations can be interpreted as coefficient of variance in raw scale.

Figure 1 displays the convergence plots for all five compartment PK parameters $\left(V_{1}, k_{a}, k_{12}, k_{21}, k_{e}\right)$. The $\mathrm{x}$ axes are these PK parameters' domain, and the y-axes are the likelihood function (13). It appears that all these PK parameter estimates reach maximum likelihood, and we don't observe any non-identifiable parameters.

Table 2 reported the PK parameter estimates. $V_{1}=33$ $\mathrm{L}, k_{a}=0.681 / \mathrm{h}, k_{12}=0.331 / \mathrm{h}, k_{21}=0.271 / \mathrm{h}$, and

Table 1 Summary of Published Non-Compartment Model Midazolam Pharmacokinetics Parameters

\begin{tabular}{ccc}
\hline Non-Compartment PK Parameters & Reported & Missed \\
\hline $\mathbf{C}_{\max }$ & 9 & 1 \\
AUC & 10 & 0 \\
$\mathbf{T}_{\max }$ & 7 & 3 \\
$\mathbf{T}_{\mathbf{1 / 2} \text {,fast }}$ & 2 & 8 \\
$\mathbf{T}_{\mathbf{1 / 2} \text {,slow }}$ & 8 & 2 \\
$\mathbf{V}_{\mathbf{d}}$ & 5 & 5 \\
$\mathrm{CL}_{\text {iv }}$ & 4 & 6 \\
\hline
\end{tabular}

There are totally 10 studies available from publications. This table shows the number of reported and missed records for the sample means of non-compartment PK parameters among those 10 studies. $k_{e}=0.67(1 / \mathrm{h})$. Please notice that $V_{1}$ has very small between study variances, $\mathrm{CV}=10 \% ; k_{a}$ has high between study variance, $\mathrm{CV}=84 \%$; and $k_{e}$ 's variation is moderate, $\mathrm{CV}=23 \%$. On the other hand, the within-study variation of reported non-compartment PK parameters is moderate, $\mathrm{CV}=27 \%$.

\section{Simulation Studies \\ Simulation Schemes}

The primary concern of this non-compartment PK parameter transformation to compartment model PK parameter is the bias of PK parameter estimates. Two simulation studies were designed to investigate this problem. In the first simulation, every non-compartment PK parameter was observed for each study. In the second simulation, the same amount of missing data as our MDZ example was assumed to be present.

In each simulation, 1000 simulated data sets were generated. Each data set had 10 studies, and each study reported either all $\left(C_{\max }, A U C, T_{1 / 2, \text { slow }}, T_{1 / 2, \text { fast }}, V_{d}\right.$, $\left.C L_{i v}\right)$ in simulation 1 , or a partial amount of $\left(C_{\max }\right.$, AUC, $\left.T_{1 / 2, \text { slow }}, T_{1 / 2, \text { fast }}, V_{d}, C L_{i v}\right)$ in simulation 2. These non-compartment model PK parameters were simulated based on the two-compartment model transformation relationship (5) and (6), their meta-analysis multivariate nonlinear mixed model (9) and (10), and MDZ PK parameter estimates and variances from Table 2.

\section{Simulation Evaluation Criteria}

Both fixed effect and variance components were evaluated in the simulation studies. The bias was calculated as the relative bias: abs(true-est)/est; and their 95\% coverage probabilities were also reported based on model based $95 \%$ confidence interval. Coverage probabilities outside of $(92.93,97.07)$ were highlighted. The halfwidth of this interval is three times the binomial stand error, which is $[(95 \%)(5 \%) / 1000]^{1 / 2}=0.6892 \%$. Standard error was also reported based on 1000 simulation results.

\section{Simulation 1 (All Reported and No Missing Data)}

Table 3 reported the simulation results. Among fixed effects, all of the relative biases are less than $1 \%$. $\left(V 1, k_{a}\right.$, $\left.k_{e}\right)$ had lower $95 \%$ coverage probabilities than $\left(K_{12}, K_{21}\right)$ did, because $\left(V_{1}, k_{a}, k_{e}\right)$ were assumed to have between study variances, but $\left(K_{12}, K_{21}\right)$ didn't have. Therefore, the low $95 \%$ coverage probability was probably due to the under estimated standard error. On the other hand, the biases of between study variance estimates were between $5 \%$ and $12.8 \%$, though their $95 \%$ CP were all around $95 \%$.

\section{Simulation 2 (With Missing Data)}

Table 4 reported the simulation results. Among fixed effects, all of the relative biases can be as high as $2.84 \%$ (i.e. $k_{e}$ ). All of their $95 \%$ coverage probabilities were outside of the normal range, $(92.93 \%, 97.07 \%)$. The low 


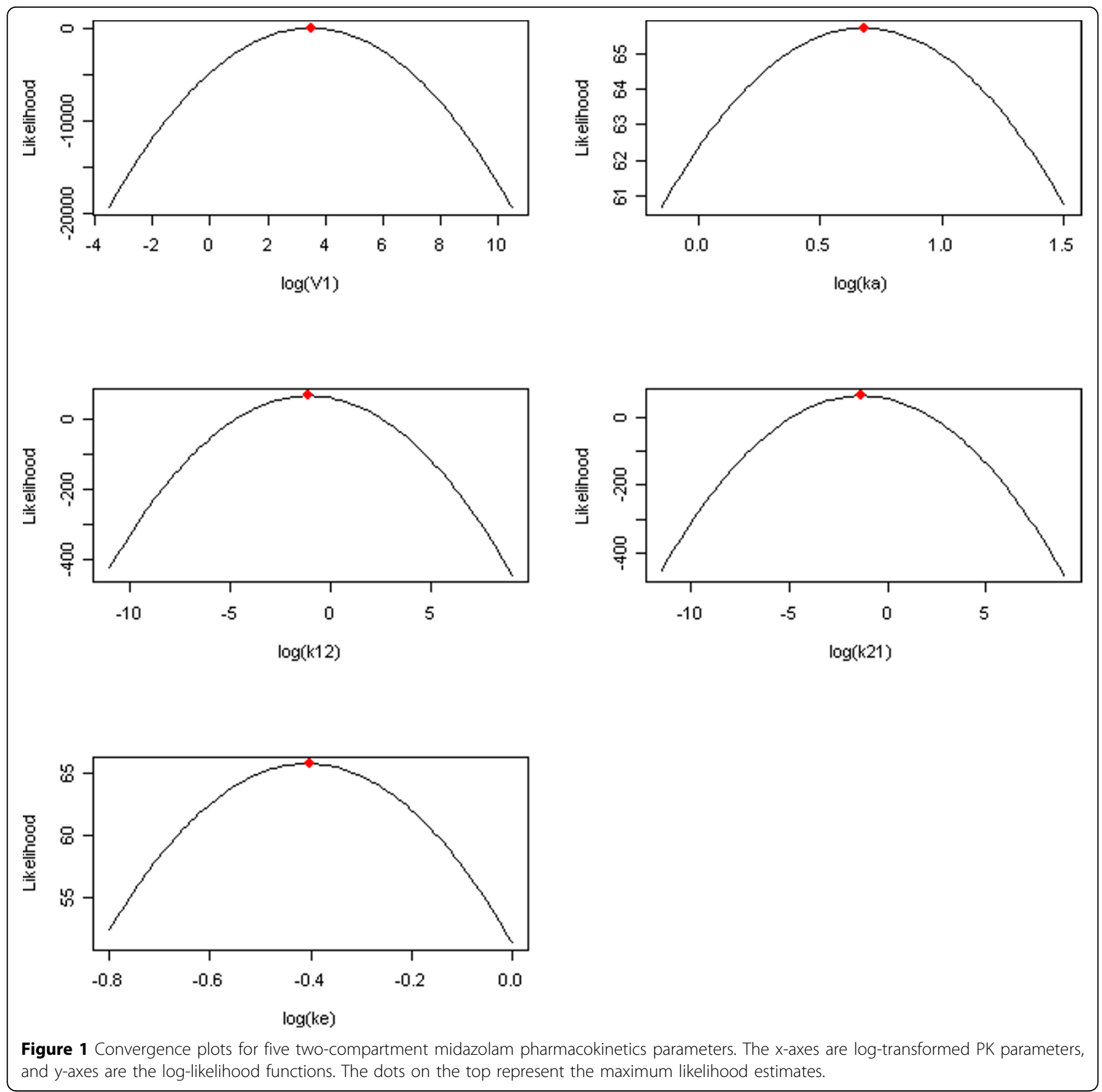

Table 2 Midazolam Compartment Model Pharmacokinetics Parameter Estimates

\begin{tabular}{cccc}
\hline Compartment Model PK Parameters & \multicolumn{2}{c}{ Non-Compartment Model to Compartment Model Transformation } \\
\cline { 2 - 4 } & \multicolumn{2}{c}{ Fixed-Effect } & raw-scale \\
Between Study CV* \\
\hline$V_{1}$ & log-scale & 33.11 & $10 \%$ \\
$k_{a}$ & 3.5 & 1.97 & $84 \%$ \\
$k_{12}$ & 0.68 & 0.33 & - \\
$k_{21}$ & -1.1 & 0.27 & - \\
$k_{e}$ & -1.32 & 0.67 & $23 \%$ \\
\hline Within-Study CV** & -0.403 & $27 \%$ & \\
\end{tabular}

These compartment model PK parameters are estimated from reported non-compartment model PK parameters. *These are between-study variances for compartment model PK parameters. ${ }^{*}$ This is the within-study variance for all non-compartment PK parameters. 
Table 3 Simulation Results with No Missing Data

\begin{tabular}{|c|c|c|c|c|c|c|}
\hline & & \multirow[b]{2}{*}{ TRUE(log-scale) } & \multicolumn{2}{|c|}{ Estimate } & \multirow[b]{2}{*}{ RelativeBias (\%) } & \multirow[b]{2}{*}{$95 \% \mathrm{CP}$} \\
\hline & & & Mean & SE & & \\
\hline & $V_{1}$ & 3.5 & 3.505 & 0.110 & 0.14 & 0.89 \\
\hline & $k_{a}$ & 0.68 & 0.680 & 0.115 & 0.02 & 0.87 \\
\hline \multirow[t]{4}{*}{ Fixed-Effect } & $k_{e}$ & -0.403 & -0.397 & 0.112 & 0.75 & 0.89 \\
\hline & $k_{12}$ & -1.1 & -1.097 & 0.088 & 0.24 & 0.93 \\
\hline & $k_{21}$ & -1.32 & -1.322 & 0.053 & 0.15 & 0.99 \\
\hline & $V_{1}$ & 0.09 & 0.083 & 0.045 & 8.05 & 0.97 \\
\hline \multirow[t]{2}{*}{ Between Study Variance } & $k_{a}$ & 0.09 & 0.078 & 0.053 & 12.8 & 0.93 \\
\hline & $k_{e}$ & 0.09 & 0.085 & 0.045 & 5.33 & 0.96 \\
\hline Sigma $^{2}$ & & 0.01 & 0.01 & 0.003 & 4.43 & 0.95 \\
\hline
\end{tabular}

Table 4 Simulation Results with Missing Data

\begin{tabular}{|c|c|c|c|c|c|c|}
\hline & & \multirow[b]{2}{*}{ TRUE(log-scale) } & \multicolumn{2}{|c|}{ Estimate } & \multirow[b]{2}{*}{ RelativeBias (\%) } & \multirow[b]{2}{*}{$95 \% \mathrm{CP}$} \\
\hline & & & Mean & SD & & \\
\hline & $V_{1}$ & 3.5 & 3.494 & 0.129 & 0.17 & 0.92 \\
\hline & $\mathrm{k}_{\mathrm{a}}$ & 0.68 & 0.672 & 0.159 & 1.13 & 0.87 \\
\hline \multirow[t]{4}{*}{ Fixed-Effect } & $k_{e}$ & -0.403 & -0.389 & 0.141 & 2.84 & 0.90 \\
\hline & $k_{12}$ & -1.1 & -1.09 & 0.172 & 0.59 & 0.84 \\
\hline & $k_{21}$ & -1.32 & -1.323 & 0.070 & 0.19 & 0.99 \\
\hline & $\mathrm{V} 1$ & 0.09 & 0.081 & 0.052 & 9.82 & 0.98 \\
\hline \multirow[t]{2}{*}{ Between Study Variance } & ka & 0.09 & 0.082 & 0.066 & 9.43 & 0.95 \\
\hline & ke & 0.09 & 0.087 & 0.055 & 3.60 & 0.97 \\
\hline Sigma $^{2}$ & & 0.01 & 0.01 & 0.003 & 13.9 & 0.96 \\
\hline
\end{tabular}

coverage of $\left(V_{1}, k_{a}, k_{e}\right)$ was probably due to their between subject variations; and the low coverage probability for $k_{12}$ and high coverage for $k_{21}$ were probably due to the missing data. As in the MDZ example, $T_{1 / 2}$, fast had only 2 out of 10 papers published. On the other hand, the biases of between study variance estimates were between $3.6 \%$ and $9.8 \%$, though their 95\% CP were within $(92.93 \%, 97.07 \%)$.

\section{Conclusions}

This paper proposed an important approach to transform published non-compartment model pharmacokinetics parameters into compartment model PK parameters. This meta-analysis was performed with a multivariate nonlinear mixed model. A conditional first-order linearization approach was developed for statistical estimation and inference, and it was implemented in R. Using MDZ as an example, we have shown that this approach transformed 6 non-compartment model PK parameters from 10 publications into 5 compartment model PK parameters, and the conditional first order linearization approach converged to the maximum likelihood. In the follow-up simulation studies, we have shown that our meta-analysis multivariate nonlinear mixed model had little relative bias $(<1 \%)$ in estimating compartment model PK parameters if all non-compartment PK parameters were reported in every study. If there existed missing non-compartment PK parameters, the relative bias of compartment model PK parameter was still small $(<3 \%)$. The $95 \%$ coverage probabilities of these PK parameter estimates were usually above $85 \%$ or more. Therefore, this approach possesses adequately valid inference.

Although this paper only showed the transformation performance of non-compartment model PK parameters to two-compartment model with oral dose PK parameters, we think it is probably the most complicated case among published drug PK studies. One compartment models and two-compartment model with IV dose have simpler transformation function and less computational expense.

Sometimes, not all of the required non-compartment model PK parameters are available in the literature. Whether it is feasible to transform these data into compartment model is an interesting and important question. In this paper, MDZ was chosen as an example. Because MDZ has been a well studied probe drug, its published non-compartment model PK parameters were expected to be rich. Other rarely studied drugs may not have all these published information, and their compartment model developments from literature need further investigations. 


\section{List of Abbreviation}

AUC: area under the concentration curve; MDZ: Midazolam; PK: Pharmacokinetics.

\section{Acknowledgements}

Dr. Lang Li is supported by NIH grants, R01 GM74217. Dr. Seongho Kim is partially supported by DOE grants, DE-EMO000197, and an Intramural Research Incentive Grant from the University of Louisville.

This article has been published as part of BMC Systems Biology Volume 4 Supplement 1, 2010: Proceedings of the ISIBM International Joint Conferences on Bioinformatics, Systems Biology and Intelligent Computing (IJCBS). The full contents of the supplement are available online at http:// www.biomedcentral.com/1752-0509/4?issue=S1.

\section{Author details}

'Division of Biostatistics, Department of Medicine, School of Medicine, Indiana University, Indianapolis, IN, 46032, USA . 'Department of Biostatistics, School of Public Health, University of Michigan, Ann Arbor, Ml, 48109, USA

\section{Authors' contributions}

ZW developed the theory of multivariate nonlinear mixed effect model, and run the implementation; SK developed the theory of multivariate nonlinear mixed effect model; SKQ provided the MDZ example background; JZ integrated the compartment model non-compartment model transformation formulas; LL initialized the idea, and developed the model transformation schemes, confirmed the statistical theory, and wrote the paper.

\section{Authors' information}

ZW is currently a Ph.D. Computer Science student in the Indiana University; SK is an assistant professor in the University of Louisville; SKQ is an assistant professor in the Indiana University; JZ is a PhD student in the University of Michigan; and LL is an association professor in the Indiana University.

\section{Competing interests}

The authors declare that they have no competing interests.

Published: 28 May 2010

\section{References}

1. DiMasi JA, Hansen RW, Grabowski HG: The price of innovation: new estimates of drug development costs. Journal of Health Economics 2003, 22(2):151-185.

2. Kola I, Landis J: Can the pharmaceutical industry reduce attrition rates? Nature Reviews Drug Discovery 2004, 3(8):711-715.

3. Woosley RL: Drug development and the FDA's critical path initiative. Clinical Pharmacology and Therapeutics 2007, , 81: 129-133.

4. Veit M: New strategies for drug development. Berl. Munch. Tierarztl. Wochenschr, 117: 276-287.

5. D'Andrea $\mathrm{G}$ e a: A polymorphism in the VKORC1 gene is associated with an interindividual variability in the dose-anticoagulant effect of warfarin. Blood 2005, , 105: 645-649.

6. Kirchheiner JB: J. Clinical consequences of cytochrome P450 2C9 polymorphisms. Clin. Pharmacol. Ther 2005, , 77: 1-16.

7. Badagnani I e a: Interaction of methotrexate with organic-anion transporting polypeptide $1 \mathrm{~A} 2$ and its genetic variants. J. Pharmacol. Exp. Ther, 318: 521-529.

8. Hung S I e a: Genetic susceptibility to carbamazepine-induced cutaneous adverse drug reactions. Pharmacogenet. Genom 2006, , 16: 297-306.

9. Cleary JD, Taylor JW, Chapman SW: Itraconazole in antifungal therapy. Ann Pharmacother 1992, 26(4):502-9.

10. Yamazaki M, Nishigaki R, Suzuki $H$, Sugiyama Y: [Kinetic analysis of hepatobiliary transport of drugs, importance of carrier-mediated transport]. Yakugaku Zasshi 1995, 115(12):953-77.

11. Okuda H, Nishiyama T, Ogura K, Nagayama S, Ikeda K, Yamaguchi S, Nakamura $Y$, Kawaguchi $Y$, Watabe T: Lethal drug interactions of sorivudine, a new antiviral drug, with oral 5-fluorouracil prodrugs. Drug Metab Dispos 1997, 25(5):270-3.

12. Lalonde RL, Hutmacher MM, Ewy W, Nichols DJ, Milligan PA, Corrigan BW, Lockwood PA, Marshall SA, Benincosa LJ, Tensfeldt TG, Parivar K, Amantea M, Glue P, Koide $H$, Miller R: Model-based drug development. Clin Pharmacol Ther 2007, 82(1):21-32.
13. Chang MKS, Bull J, Chiu YY, Wang W, Wakeford C, McCarthy K: Innovative approaches in drug development. J Biopharm Stat 2007, 17(5):775-89.

14. RT ON: FDA's critical path initiative: a perspective on contributions of biostatistics. Biom J 2006, 48(4):559-64.

15. Chien JY, Heathman MA, de Alwis DP, Sinha V: Pharmacokinetics/ Pharmacodynamics and the stages of drug development: role of modeling and simulation. The AAPS Journal 2005, 7(3):E544-E559.

16. Rowland M, Tozer TN: Clinical Pharmacokinetics Concept and Applications. Lippincott Williams \& Wilkins, Third 1995.

17. Davidian M, Giltinan DM: Nonlinear models for repeated measurment data. Chapman and Hall 1995.

18. Wakefield JC, Rahman N: The combination of population pharmacokinetic studies. Biometrics 2000, 56(1):263-70.

19. Lopes HF, Muller P, Rosner GL: Bayesian meta-analysis for longitudinal data models using multivariate mixture priors. Biometrics 2003, 59(1):66-75.

20. Li L, Yu M, Chin R, Lucksiri A, Flockhart D, Hall S: Drug-Drug Interaction Prediction: A Bayesian Meta-Analysis Approach. Statistis in Medicine 2007, 26(20):3700-3721.

21. Yu M, et al: A Bayesian meta-analysis on published sample mean and variance pharmacokinetic data with application to drug-drug interaction prediction. J Biopharm Stat 2008, 18(6):1063-83.

22. Wolfinger RD: Two Taylor-series approximations methods for nonlinear mixed models. Journal of Computational Statistics and Data Analysis 1997, 25:465-490.

23. Wolfinger R, O'Connell M: Generalized linear mixed models: a pseudolikelihood approach. Journal of statistical Computation and Simulation 1993, 48:233-243.

24. Lindstrom ML, Bates DM: Nonlinear mixed effects models for repeated measures data. Biometrics 1990, 46(3):673-87.

25. Wolfinger RD: Laplace's Approximation for Nonlinear Mixed Models. Biometrika 1993, 80:791-795.

26. Westfall P, Hochberg Y, Wolfinger RD, Rorn D, Tobias RD: Multiple comparisons and Multiple Tests. 1999.

27. Vonesh EF: A Note on Laplace's Approximation in Nonlinear Mixed Effects Models. Biometrika 1996, 83:447-452.

doi:10.1186/1752-0509-4-S1-S8

Cite this article as: Wang et al:: Non-compartment model to compartment model pharmacokinetics transformation meta-analysis - a multivariate nonlinear mixed model. BMC Systems Biology 2010 4(Suppl 1):S8.

\section{Submit your next manuscript to BioMed Central and take full advantage of:}

- Convenient online submission

- Thorough peer review

- No space constraints or color figure charges

- Immediate publication on acceptance

- Inclusion in PubMed, CAS, Scopus and Google Scholar

- Research which is freely available for redistribution 